\title{
COMMENTARY
}

\section{Hot times in the intensive care unit}

\author{
Edward Abraham* \\ See related research by Morris et al., http://ccforum.com/content/14/3/R125
}

\begin{abstract}
Fever is a common occurrence in the intensive care unit, and pharmacologic approaches are limited, particularly in patients unable to tolerate enteral medications. Although a study by Morris and colleagues in the previous issue of Critical Care suggests that intravenous ibuprofen is safe and effective in critically ill patients, the study is small and the drug was given over only a 24-hour period. Additional studies will need to be performed to demonstrate the safety and efficacy of intravenous ibuprofen in critically ill patients.
\end{abstract}

Fever in the context of critical illness is a frequent occurrence and can raise the concern that an infection or other inflammatory process, such as pancreatitis, is present. In this situation, the evaluative process for the cause of fever may result in important modifications in treatment, including initiation or alteration in antimicrobial therapy. Fevers can also be a manifestation of drug reactions, prompting the discontinuation of suspected agents. However, in many cases, fever in a critically ill patient is not associated with a treatable etiology and is considered simply a symptom that accompanies the organ dysfunction that brought the patient to the intensive care unit (ICU).

The optimal approach to fever in the ICU has not been resolved. While reducing fever can improve patient comfort, decrease the risk of febrile seizures in the predisposed patient, and lower metabolic rate, it remains unclear whether antipyretics actually affect patient outcome in clinically meaningful ways. Nevertheless, the use of acetaminophen (paracetamol), ibuprofen, and other oral agents remains a common practice in both outpatient and inpatient settings. However, there are limited options available for lowering temperature in critically ill

*Correspondence: eabraham@uab.edu

Department of Medicine, University of Alabama at Birmingham School of Medicine, 420 Boshell Building, 1808 7th Avenue South, Birmingham, AL 35294, USA patients in whom the enteral route cannot be used. External cooling measures have only modest effects, especially if there is peripheral vasoconstriction, and the use of invasive techniques, including chilled intravenous fluids and peritoneal or extracorporeal cooling measures, is generally not indicated for the modest hyperthermia commonly present in the ICU.

In the previous issue of Critical Care, a clinical trial reported by Morris and colleagues [1] shows that intravenous ibuprofen is more effective than placebo in lowering temperature to less than $101^{\circ} \mathrm{C}$ in both critically ill and non-critically ill patients. Although there did not appear to be an increased incidence of adverse events in the patients who received intravenous ibuprofen, this study was small, with just 53 critically ill patients, and specifically excluded groups of patients commonly seen in the ICU, such as those immediately post-surgery, those with creatinine of greater than $3 \mathrm{mg} / \mathrm{dL}$, or those receiving dialysis or corticosteroid therapy. Therefore, claims of safety for the use of intravenous ibuprofen in critically ill patients need to be well established with larger studies before such therapy can be considered for routine use.

Concerns about the use of intravenous ibuprofen in the ICU are further magnified by the lack of any suggestion of clinical benefit with such therapy. Although a study by Bernard and colleagues [2] showed that intravenous ibuprofen was effective in diminishing the severity of hyperthermia in patients with acute lung injury, there did not appear to be any beneficial effects on survival or other meaningful clinical outcomes.

The safety concerns with the use of ibuprofen in critically ill patients should not be underestimated. Even in healthy patients, ibuprofen and similar nonsteroidal anti-inflammatory agents are associated with renal dysfunction, particularly in patients with baseline evidence of renal compromise [3]. Owing to renal hypoperfusion associated with sepsis, hypovolemia, or diminished cardiac output or in response to nephrotoxic drugs, such as aminoglycosides, which are frequently used in critically ill patients, acute kidney insufficiency is a common occurrence in the ICU. In the study by Morris and colleagues [1], intravenous ibuprofen was administered over only a 24-hour period. As fever is often a 
persistent problem in critically ill patients, occurring over many days, the potential use of intravenous ibuprofen is unlikely to be limited to only 24 hours. Although the present study appears to show that a very limited exposure to intravenous ibuprofen in patients without evidence of significant renal dysfunction is not associated with significant harm, we really need to be assured of the safety of this intervention over a more prolonged period and in more typical ICU populations before its routine use can be considered.

The question of whether we should be treating fever at all remains. Although there may be some reason to believe that massive and prolonged hyperpyrexia is harmful, there is little evidence that the more modest fevers commonly seen in ICU patients affect their clinical outcomes. Until we have such data, there seems to be little use in treating fever at all, especially as the development of fever may provide an important clue alerting us to the need for modifying therapy for pathophysiologic processes, such as a new infection, that really can determine the outcome for a patient.
Abbreviation

ICU, intensive care unit.

\section{Competing interests}

The author declares that he has no competing interests.

Published: 19 July 2010

\section{References}

1. Morris PE, Promes JT, Guntupalli KK, Wright PE, Arons MM: A multi-center, randomized, double-blind, parallel, placebo-controlled trial to evaluate the efficacy, safety, and pharmacokinetics of intravenous ibuprofen for the treatment of fever in critically ill and non-critically ill adults. Crit Care 2010, 14:R125.

2. Bernard GR, Wheeler AP, Russell JA, Schein R, Summer WR, Steinberg KP, Fulkerson WJ, Wright PE, Christman BW, Dupont WD, Higgins SB, Swindell BB: The effects of ibuprofen on the physiology and survival of patients with sepsis. The Ibuprofen in Sepsis Study Group. N Engl J Med 1997, 336:912-918.

3. Amer M, Bead VR, Bathon J, Blumenthal RS, Edwards DN: Use of nonsteroidal anti-inflammatory drugs in patients with cardiovascular disease: a cautionary tale. Cardiol Rev 2010, 18:204-212.

doi:10.1186/cc9092

Cite this article as: Abraham E: Hot times in the intensive care unit. Critical Care 2010, 14:178. 